\title{
Nasal transcriptome and epigenome analysis identifies the pathogenic features of aspirin-exacerbated respiratory disease
}

\author{
Ga-Young Ban ${ }^{1}$, Eun-Kyung Kwon ${ }^{1}$, Tae-Wook Kang ${ }^{2}$, Taeyun $\mathrm{Oh}^{3}$, Oak-Sung Choo ${ }^{4}$, \\ Young-Min $\mathrm{Ye}^{5}$, and Hae-Sim Park ${ }^{5}$ \\ ${ }^{1}$ Hallym University College of Medicine \\ ${ }^{2}$ Daejeon University \\ ${ }^{3}$ Yonsei University College of Medicine Department of Internal Medicine \\ ${ }^{4}$ Nowon Eulji University Hospital \\ ${ }^{5}$ Ajou University
}

January 23, 2022

\begin{abstract}
Background: Dysregulation of the arachidonic acid metabolic pathway is the most widely known pathomechanism of AERD. We performed integrative analysis of transcriptomic and epigenomic profiling with network analysis to determine the novel pathogenic features of AERD. Methods: Ten patients with asthma including 5 patients with AERD and another 5 patients with aspirin tolerant asthma (ATA) were enrolled. Nasal epithelial scraping was performed and nasal mucosa was used in omics profiling. Peripheral eosinophil counts, sputum eosinophil counts, FeNO levels, and pulmonary function test results were evaluated. Differentially expressed genes (DEGs), differentially methylated probes (DMPs) and differentially correlated genes (DCGs) between patients with AERD and those with ATA were analyzed. Network analysis using Ingenuity Pathway Analysis (IPA) was performed to determine the gene connection network and signaling pathways. Results: In total, 1,736 DEGs and 1,401 DMPs were identified. Finally, 19 pairs for DCGs were selected. Among DCGs, genes related to vesicle transport (e.g. STX2 and $R A B 3 B$ ) and sphingolipid dysregulation (e.g. SMPD3) were found to be hypo-methylated and up-regulated in patients with AERD. A total number of 78 asthma-related DEGs were identified by the IPA knowledge base. Using the canonical pathway analysis of IPA, signaling pathways of $\mathrm{T}$ helper cell differentiation/activation and Fce receptor I were generated.

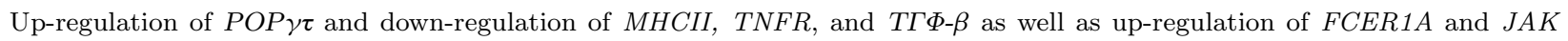
and down-regulation of $V A V$ and $c P L A 2$ were noted in patients with AERD. Conclusions: Distinct pathogenic features were identified by using integrative multi-omics data analysis in patients with AERD.
\end{abstract}

\section{Introduction}

Aspirin-exacerbated respiratory disease (AERD) is characterized by clinical features of persistent asthma, sinonasal inflammation with nasal polyposis, and hypersensitivity reactions to cyclooxygenase- 1 inhibitors. ${ }^{1}$ Patients with AERD are known to have more severe airway obstruction and frequent asthma exacerbation than those with aspirin tolerant asthma (ATA). A recent report has suggested that the prevalence of AERD is $7 \%$ in adult asthmatics, while $14 \%$ in severe asthmatics. ${ }^{2}$

Immunological pathophysiology of AERD is not fully understood; however, a number of studies have provided evidence that numerous kinds of immune cells (eosinophils, mast cells, innate lymphoid cells, and platelet-adherent leukocytes) and mediators (leukotriene E4, prostaglandin E2, prostaglandin D2, IL-25, and IL-33) are involved. ${ }^{3}$ Dysregulation of arachidonic acid metabolism is a well-known pathophysiology in AERD. Reduced levels of prostaglandin E2, increased levels of prostaglandin D2 and leukotriene E4 play important roles in airway inflammation and bronchoconstriction in AERD. ${ }^{4}$ In this context, the level of urinary 
leukotriene $\mathrm{E} 4$ has been proposed as a potential biomarker to determine $\mathrm{AERD}$, and leukotriene receptor antagonists (e.g. montelukast or pranlukast) are widely used in AERD treatment. ${ }^{5,6}$ Meanwhile, the levels of leukotriene $\mathrm{E} 4$ were reported to be remained high with increased eosinophil inflammation and impaired asthma control despite of the leukotriene receptor antagonist treatment in AERD patients. ${ }^{7}$ To attenuate the activation status of eosinophils, mast cells and innate lymphoid cells in AERD pathogenesis, previous studies have suggested anti-IL4R (e.g. dupilumab), anti-IL-5 (e.g. mepolizumab or reslizumab), anti-IgE (e.g.omalizumab), and anti-IL-33/TSLP antibody treatment of severe type 2 asthma in addition to inhaled corticosteroids with long-acting beta 2 -agonists. ${ }^{1,8}$ Although these biologics are closely related to pathologic mediators in AERD, the high medical cost and incomplete blockage of non-steroidal anti-inflammatory drug-induced reactions should be carefully considered.

To identify novel causal pathways and therapeutic targets of AERD, we performed RNA and methylation sequencing using nasal epithelial scrapings from AERD patients as compared to ATA patients.

\section{Materials and Methods}

\section{Study subjects and sample collection}

Five patients with AERD and another 5 patients with ATA were enrolled at Ajou University Hospital in Suwon, South Korea. Asthma was diagnosed according to the Global Initiative for Asthma guideline (GINA) 2021 by the allergy specialists. AERD was defined by a recurrent clinical history (exacerbation of upper or lower respiratory reactions after ingestion of aspirin/NSAIDs) and/or a positive response to the lysine-aspirin bronchial provocation test (Lys-ASA BPT). The Lys-ASA BPT was performed with increasing doses of Lys-ASA solution up to $300 \mathrm{mg} / \mathrm{mL}$ using the method previously reported. ${ }^{9}$ The positive result of the Lys-ASA BPT was defined when $\mathrm{FEV}_{1} \%$ was decreased by more than $20 \%$ after the challenge. Asthmatics with negative results to the Lys-ASA BPT or denied any changes in upper or lower respiratory tract symptoms after ingestion of aspirin/NSAIDs were defined as ATA. Severe asthma was defined as the international ERS/ATS guidelines. ${ }^{10}$ Exclusion criteria for enrollment were as follows: 1) asthmatics who had been treated with type 2 biologics, including omalizumab, mepolizumab, reslizumab, benralizumab, and dupilumab, within 130 days of enrollment; 2) current smokers or ex-smokers who quit smoking within 30 days of enrollment; and 3) asthmatics who used any of intranasal corticosteroids, intranasal antihistamines, and intranasal anticholinergics within 7 days of enrollment.

Nasal scraping was performed with a pencil-shaped disposable nasal curette (Rhino-probe ${ }^{\circledR}$, Arlington Scientific, Inc., Springville, UT, USA) for 3 times from the middle portion of the each side of the inferior turbinate after 2 nasal lavages (10-mL saline each) to remove overlying mucus. Peripheral eosinophil counts, sputum eosinophil counts, total immunoglobulin E ( $\mathrm{IgE}$ ), fractional exhaled nitric oxide (FeNO) levels, pulmonary function test results, and atopy status were evaluated at enrollment. All subjects gave written informed consent at the time of enrolment, and the study was approved by the Institutional Review Board of Ajou University Hospital (AJIRB-BMR-SUR-17-182).

\section{RNA sequencing and data analysis}

Total RNA was also extracted from nasal mucosa tissues using a Nucleo spin RNA kit (MACHREY-NAGEL, Dueren, Germany). The quantity and quality of total RNA were evaluated using an Agilent 2100 Bioanalyzer (Agilent, Santa Clara, CA, USA) and a RNA quality indicator (RIN). Library preparation was conducted with an Illumina TruSeq Stranded mRNA sample preparation kit (Illumina, San Diego, CA, USA) according to the manufacturer's instructions. Indexing adapters were ligated to the ends of the cDNA fragments using ligation mix reagent at $30 \mathrm{degC}$ for $10 \mathrm{~min}$. After twice washing with sample purification beads, PCR was performed to enrich those cDNA fragments that have adapter molecules on both ends. The quality and band size of the libraries were assessed using an Agilent 2100 Bioanalyzer. Libraries were quantified by qPCR using the CFX96 Real Time System (Biorad, Hercules, CA, USA). Sequencing of the prepared library was conducted on the Hiseq2500 platform (Illumina) with 100 bp paired-end reads.

Reads were trimmed to remove adapters and low-quality reads (per-base quality $<20$ ) and thereby improve 
paired-end mapping. High-quality sequence reads were mapped to the Human genome (hg19) using STAR, ${ }^{11}$ and gene expression levels were quantified with the RSEM (v. 2.12.0). ${ }^{12}$ Differentially expressed genes (DEGs) between the 2 groups were evaluated by the edgeR package (v. 3.0.8), ${ }^{13}$ which is based on negative binomial models for RNA-seq count data. DEGs were screened with a cutoff threshold of $\log (\mathrm{FC})[?]|1|$ and $P$ value $<0.05$.

\section{Methylation assay and data analysis}

Genomic DNA was also extracted from nasal mucosal tissues by using the RNA-seq using Illumina Infinium Methylation EPIC Bead Chip kits (Illumina, Inc.), and arrays were scanned by the Standard Illumina procedures using an Illumina iScan scanner. Each methylation data point is represented by fluorescent signals from the M (methylated) and $\mathrm{U}$ (unmethylated) alleles. Background intensity computed from a set of negative controls was subtracted from each analytical data point. The ratio of fluorescent signals was then computed from the 2 alleles ss $=(\max (\mathrm{M}, 0)) /(|\mathrm{U}|+|\mathrm{M}|+100)$. The ss-value reflects the methylation level of each CpG site. A ss-value of 0-1 was reported signifying percent methylation, from $0 \%$ to $100 \%$, for each CpG site.

After methylation assay, array data export processing and analysis were performed using Illumina GenomeStudio v2011.1 (Methylatioin Module v1.9.0) and R 3.0.2 (http://www.r-project.org). Differentially methylated probes (DMP) and regions were detected through the in house python scripts using the numpy and scipy package $^{14}$ and ChAMP $^{15}$ with a cutoff threshold of $P$ value $<0.001$.

\section{Correlation analysis between RNA expression and DNA methylation}

Correlation analysis was performed to identify genes regulated by DNA methylation. To identify differentially correlated genes (DCG) which show an inverse relationship between expression and methylation, Spearman rank correlations were used to assess the relationship between $\mathrm{CpG}$ methylation and gene expression. Rpackage was used for statistical analysis

\section{Pathway and network analysis}

Ingenuity pathway analysis (IPA, Redwood City, CA, USA) was used to understand the functional characteristics of DEGs of AERD. ${ }^{16}$ IPA canonical pathway analysis was performed with DEGs to identify biological pathways that significantly affect mRNA expression in AERD. The gene symbols of probe, which significantly up-regulated or down-regulated in AERD $(\log (\mathrm{FC})[?]|1|$ and $P$ value $<0.05)$ compared to ATA were selected as significantly differentiated genes and used for IPA analysis. The significance of the association between the data set and the canonical pathways was measured as the number of molecules in each pathway that meets cutoff criteria (false discovery rate (FDR)-adjusted $P$ values $<0.05$ ).

Network analysis was performed using asthma-related DEGs to understand and predict interactions among these genes. The biological functions that were significantly associated with the genes in the core networks were identified by functional analysis based on the Ingenuity's knowledge base. As our study is focused on asthma population, asthma-related terms were selected and used in further analysis. We determined asthma-related terms such as refractory asthma, severe asthma, uncontrolled asthma, eosinophilia of lung, pulmonary eosinophilia, T helper 2 (Th2)-mediated asthma, and aspirin-induced asthma. The DEGs were mapped onto the networks to explore the dynamic changes during the asthma process.

\section{Results}

\section{Characteristics of the study subjects}

Table 1 shows the clinical characteristics of the study subjects. The sputum eosinophil counts were higher in AERD patients than in ATA patients $(P=0.032)$. Otherwise, no significant differences were noted between patients with AERD and those with ATA.

Expression profile and identification of DEGs between patients with ATA and those with AERD 
From the 10 asthmatic patients collected, the average 35,160,551 pairs of RNA-seq per sample was produced, and $35,115,725$ pairs $(7,039,115,605 \mathrm{bp})$ were presented after the adapter and low quality trimming (Table S1). Gene expression profiling was conducted by reads mapping against the human reference genome (hg19), and the normalization and sample clustering was attempted to compare between the AERD and ATA groups (Figure $1 \mathrm{~A}$ and Table S2).

DEGs were selected according to aspirin intolerance, adjusting with atopy status. Under the criteria of $P$ value $<0.05$ and $\log$ FC [?] |1|; we found 1,736 DEGs: 832 were up-regulated and 904 were down-regulated in patients with AERD (Figure 1, B and C).

Estimation of methylation levels and D ifferentiallyM ethylated $\mathbf{R}$ egions (DMRs)

The number of statistically significant CpGs from the chip was 865,912 at the $P$ value $<0.01$ and 865,917 at the $P$ value $<0.05$. Of the total samples (10 patients), $\mathrm{CpG}$ sites whose uncertain methylation values were greater than $25 \%$ in the sum of NA, and $P$ values $>0.05$ were excluded from further analysis. The remaining 865,384 CpGs after the filtering were used for subsequent analysis.

The DNA methylation profile was generated in 10 asthmatics. After adjusting for atopy status, we found 1,401 DMPs using ChAMP (R-package for methylation analysis pipeline): 13 with higher methylation and 1,388 with lower methylation in patients with AERD (Figure 2A and Table S3). As shown above, the genomic distribution of extracted DMPs was confirmed at the significance level of $P$ value and the condition of aspirin intolerance. Most DMPs from AERD and ATA were frequently observed at the upstream regulatory region, such as TSS, UTR, and 1st Exon, according to the ratio of the region occupied by whole genome (Figure $2 \mathrm{~B}$ ), suggesting that methylation changes occur primarily in the regulatory domain.

\section{Determination of DCGs (RNA and DMP)}

DCGs are genes whose expression and DNA methylation patterns significantly differ in patients with AERD compared to those with ATA. With 1,736 DEGs (832 up-regulated, 904 down-regulated) and 1,401 DMPs (897 genes), 83 pairs for DEG and its CpG which is located at the promoter of the corresponding gene were found (Table S4). Finally, 19 pairs (19 DMPs and 18 DEGs) with negative correlation and at $P<0.05$ were selected (Figure 3, Table 2), and all candidates were up-regulated and hypo-methylated in patients with AERD.

\section{Pathway analysis and gene connection network of AERD}

Results from canonical pathway analysis using all 1,736 DEGs are shown in Figure S1. As our study subjects were asthmatics, asthma-related terms were selected by the IPA knowledge base, DEGs were used to identify gene-to-gene connections in asthma-related networks. Among 78 asthma-related DEGs which are listed in Table S5, 37 were found to be connected in the network (Figure 4A). Information about 37 DEGs is provided in Table S6. Based on this gene connection network, canonical pathways, which are significantly different between patients with AERD and those with ATA, were discovered by using the IPA. The top 20 enriched canonical pathways are presented in Table S7. Of particular interest in T helper cells and mast cells, signaling pathways of $\mathrm{T}$ helper cell differentiation/activation and $\mathrm{F} c \varepsilon$ receptor I were generated (Figure 4, B and C). Up-regulation of the retinoic acid receptor-related orphan receptor $\gamma \mathrm{t}(P O P \gamma \tau)$ and down-regulation of

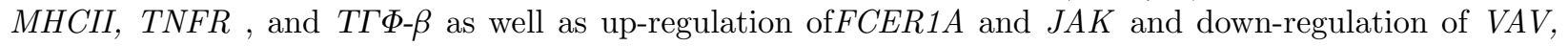
$c P L A 2$ were noted in $\mathrm{T}$ helper cell differentiation/activation as well as the Fce receptor I signaling pathway, respectively.

\section{Genes associated airway eosinophilic inflammation}

Logistic regression analysis was performed to evaluate whether the levels of specific gene expression affect airway eosinophilic inflammation adjusted for age and sex. Gene expression levels of RAB3B, SYNE1, STX2 , SMPD3 and POP $\gamma \tau$ significantly affected sputum eosinophil counts (Table S8).

\section{Discussion}


This is the first study to demonstrate the transcriptomic and epigenomic profiles of patients with AERD. To discover the distinct signature of AERD, integrated analysis of transcriptomes and epigenomes was performed with the nasal tissue from asthmatics. We identified the genes related to vesicle transport, sphingolipid regulation, $\mathrm{T}$ helper cell and mast cell activation were dysregulated in AERD. In addition, the mRNA expression levels of these genes were significantly associated with the sputum eosinophil counts. Collectively, we identified the distinct transcriptomic and epigenomic signature of AERD, which may contribute to the pathogenesis of eosinophilic inflammation of AERD.

AERD is characterized by persistent eosinophil activation and overproduction of cyteinyl leukotrienes. The most important cells involved in the pathogenesis of AERD are eosinophils and mast cells. ${ }^{17}$ These cells contribute to airway inflammation and hyper-responsiveness through the release of diverse mediators (e.g. cytokines, chemokines, and growth factors). ${ }^{18}$ Bidirectional cytokine release induces reciprocal activation of eosinophils, mast cells, Th2 cells, and group 2 innate lymphoid cells. Preformed mediators which are stored in intracellular granules are released to the outside of the cells by several distinct mechanisms; classic exocytosis, compound exocytosis, piecemeal degranulation, and cytolysis. ${ }^{19}$ This degranulation process involves multiple steps of membrane fusion. Most of the membrane fusion events are mediated by soluble $N$ -ethylmaleimide-sensitive factor attachment protein receptors (SNARE) proteins. ${ }^{20}$ The SNARE complex is originally composed of v-SNAREs (e.g. vesicle-associated membrane protein) associated with the vesicle and t-SNARE (e.g. syntaxins) associated with the target compartment. Previous studies have reported the mRNA and protein expressions of SNAREs (e.g. syntaxins or vesicle-associated membrane protein) in diverse inflammatory cells of humans. ${ }^{21}$ In the present study, we found the $S T X 2$ gene was differentially correlated between patients with AERD and those with ATA. In addition, the mRNA expression levels ofSTX2 significantly affected the sputum eosinophil counts. These findings collectively suggest that hypomethylation resulted in the up-regulation of the $S T X$ gene and then induced eosinophil inflammation in a target tissue of AERD patients.

Rab proteins, which belong to the GTP-binding protein superfamily, also participate in intracellular vesicle trafficking. They regulate the targeting/docking/fusion process of vesicle transport and the assembly of SNARE proteins. ${ }^{22,23}$ The release of granules from eosinophils, neutrophils, and platelets is facilitated by these Rab proteins. ${ }^{22,}{ }^{23}$ In a previous study, a common variant in $R A B 27 A$ has been identified to have association with FeNO levels in adults. ${ }^{24}$ Another study reported the association of single-nucleotide polymorphisms on the $R A B 1 A$ gene with the risk of AERD and with the responsiveness of airways to aspirin. ${ }^{25}$ The $R A B 1 A$ gene has been suggested to play a role in the development of AERD. ${ }^{25}$ Consistent with these previous studies, we observed the $R A B 3 B$ gene was a more significant DCG which was hypomethylated and up-regulated in patients with AERD compared to those with ATA. Furthermore, the sputum eosinophil counts were affected by the levels of mRNA expression of the $R A B 3 B$ gene. Taken together, genes related to vesicle transport were significantly hypo-methylated and up-regulated in patients with AERD, which may promote eosinophilic inflammation in a target tissue.

Dysregulation of sphingolipids in AERD and severe asthma has been reported in several studies. ${ }^{6,26-28}$ Increased levels of ceramides enhanced the release of asthma-related cytokines and chemokines in an asthma mouse model. ${ }^{29}$ Ceramides can be generated via the de novo pathway by serine palmitoyl transferase and sphingomyelinase pathways regulated by sphingomyelinases, or the recycling pathway. ${ }^{30}$ Increased levels of serine palmitoyl transferase, long-chain base subunit $2,{ }^{28}$ and $S M P D 1{ }^{31}$ have been suggested to induce ceramide increase, and augment eosinophilic inflammation in AERD. ${ }^{28,31}$ In this context, we found the SMPD 3 gene was hypo-methylated and up-regulated in patients with AERD compared to those with ATA. This finding indicates that sphingolipids may play a role in the pathogenesis of AERD, which is strengthened by previous study results.

Type 2 (T2) inflammatory responses are known to play a central role in patients with AERD. Th2 and Th17 inflammation mutually affect each other, resulting in the augmentation of T2 immune responses. ${ }^{32-34}$ An IL-17-deficient allergic asthma mice model showed significant reductions in both Th17 and Th2 immune responses. ROR $\gamma t$ is a member of the nuclear receptor superfamily that regulates Th17 differentiation. 
ROR $r$ inhibitors also diminished Th17 and Th2 immune responses in an animal model of allergic asthma. ${ }^{33}$ TGF- $\beta$ plays an important role in the development of regulatory $\mathrm{T}$ (Treg) cells. The defective regulatory function of Treg cells has been noticed in pollen-allergic subjects. ${ }^{35}$ Based on these previous studies, upregulated $P O P \gamma \tau$ and down-regulated $T \Gamma \Phi-\beta$ may play a role in the augmentation of T2 inflammation in patients with AERD.

Mast cell activation as well as release of cysteinyl leukotrienes, and prostaglandin D2 is an essential component of AERD pathogenesis. Mast cell activation is initiated by cross-linking of the high-affinity Fc receptor for IgE (FceRI) with IgE-bound antigen. ${ }^{36,}{ }^{37}$ Based on definitive evidence for mast cell activation in airway inflammation of AERD, omalizumab treatment has been reported to successfully reduce the levels of leukotrienes and eosinophils in AERD patients. ${ }^{8}$ Omalizumab, a monoclonal anti-IgE antibody, binds to circulating free IgE, disrupts FceRI: IgE complex and decreases FceRI expression, resulting in the regulation of allergic reactions. In the present study, up-regulation of the FCER1A gene expression was observed which is compatible with the results of previous studies, suggesting the role of FceRI in AERD pathogenesis.

This study has two limitations. One is that nasal tissues were used to profile the transcriptome and epigenome. Expression profiling using target tissues, such as bronchial airway epithelial cells obtained by endobronchial brushing, may have stronger power that reflects airway genetic signatures. ${ }^{38}$ However, in actual clinical practice, it is difficult to perform invasive bronchoscopy for collecting target tissues. Nasal cytology of nasal curette specimens, a simple and non-invasive procedure which allows for assessing the pathologic features of nasal mucosa, is a good alternative method. ${ }^{39,}{ }^{40}$ Moreover, strong correlations between bronchial and nasal airway gene expression profiles have been reported in previous studies. ${ }^{38,41}$ The other is that the number of the study subjects was too small to generalize the study results. Further studies are needed with a larger sample size.

In conclusion, our results demonstrated the distinct omics signature that is related to vesicle transport, sphingolipid regulation, and $\mathrm{T}$ helper cell/mast cell activation in AERD, which may play an important role in the pathogenesis of AERD.

\section{References}

1. Choi Y, Lee Y, Park H-S. Which Factors Associated With Activated Eosinophils Contribute to the Pathogenesis of Aspirin-Exacerbated Respiratory Disease? Allergy Asthma Immunol Res . 2019;11:320-329.

2. Rajan JP, Wineinger NE, Stevenson DD, White AA. Prevalence of aspirin-exacerbated respiratory disease among asthmatic patients: A meta-analysis of the literature. J Allergy Clin Immunol . 2015;135:676-681.

3. Wangberg H, White AA. Aspirin-exacerbated respiratory disease.Curr Opin Immunol . 2020;66:9-13.

4. Le Pham D, Lee JH, Park HS. Aspirin-exacerbated respiratory disease: an update. Curr Opin Pulm Med . 2017;23:89-96.

5. Hagan JB, Laidlaw TM, Divekar R, et al. Urinary Leukotriene E4 to Determine Aspirin Intolerance in Asthma: A Systematic Review and Meta-Analysis. The journal of allergy and clinical immunology In practice . 2017;5:990-997.

6. Ban GY, Cho K, Kim SH, et al. Metabolomic analysis identifies potential diagnostic biomarkers for aspirin-exacerbated respiratory disease. Clin Exp Allergy . 2017;47:37-47.

7. Ban G-Y, Kim S-H, Park H-S. Persistent Eosinophilic Inflammation in Adult Asthmatics with High Serum and Urine Levels of Leukotriene E4.J Asthma Allergy . 2021;14:1219.

8. Hayashi H, Fukutomi Y, Mitsui C, et al. Omalizumab for Aspirin Hypersensitivity and Leukotriene Overproduction in Aspirin-exacerbated Respiratory Disease. A Randomized Controlled Trial. Am J Respir Crit Care Med . 2020;201:1488-1498.

9. Park H. Early and late onset asthmatic responses following lysine-aspirin inhalation in aspirin-sensitive asthmatic patients. Clin Exp Allergy . 1995;25:38-40. 
10. Chung KF, Wenzel SE, Brozek JL, et al. International ERS/ATS guidelines on definition, evaluation and treatment of severe asthma.Eur Respir J . 2014;43:343-373.

11. Dobin A, Davis CA, Schlesinger F, et al. STAR: ultrafast universal RNA-seq aligner. Bioinformatics . 2013;29:15-21.

12. Li B, Dewey CN. RSEM: accurate transcript quantification from RNA-Seq data with or without a reference genome. BMC Bioinformatics . 2011;12:1-16.

13. Robinson MD, McCarthy DJ, Smyth GK. edgeR: a Bioconductor package for differential expression analysis of digital gene expression data.Bioinformatics . 2010;26:139-140.

14. Oliphant TE. Python for scientific computing. Computing in science $\mathscr{E}$ engineering . 2007;9:10-20.

15. Tian Y, Morris TJ, Webster AP, et al. ChAMP: updated methylation analysis pipeline for Illumina BeadChips. Bioinformatics . 2017;33:3982-3984.

16. Ingenuity Pathway Analysis Systems [cited 2021 July 10]. Available from:http://www.ingenuity.com.

17. Woo SD, Luu QQ, Park HS. NSAID-Exacerbated Respiratory Disease (NERD): From Pathogenesis to Improved Care. Front Pharmacol . 2020;11:1147.

18. Davoine F, Lacy P. Eosinophil cytokines, chemokines, and growth factors: emerging roles in immunity. Front Immunol . 2014;5:570.

19. Spencer LA, Bonjour K, Melo RC, Weller PF. Eosinophil secretion of granule-derived cytokines. Front Immunol . 2014;5:496.

20. Singh J, Shah R, Singh D. Targeting mast cells: Uncovering prolific therapeutic role in myriad diseases. Int Immunopharmacol . 2016;40:362-384.

21. Woska JR, Jr., Gillespie ME. SNARE complex-mediated degranulation in mast cells. J Cell Mol Med . 2012;16:649-656.

22. Shukla A, Berglund L, Nielsen L, et al. Regulated exocytosis in immune function: are SNARE-proteins involved? Respir Med . 2000;94:10-17.

23. Takai Y, Sasaki T, Matozaki T. Small GTP-binding proteins.Physiol Rev . 2001;81:153-208.

24. Bouzigon E, Nadif R, Thompson EE, et al. A common variant in RAB27A gene is associated with fractional exhaled nitric oxide levels in adults. Clin Exp Allergy . 2015;45:797-806.

25. Park JS, Heo JS, Chang HS, et al. Association analysis of member RAS oncogene family gene polymorphisms with aspirin intolerance in asthmatic patients. DNA Cell Biol . 2014;33:155-161.

26. Reinke SN, Gallart-Ayala H, Gomez C, et al. Metabolomics analysis identifies different metabotypes of asthma severity. Eur Respir J . 2017;49.

27. Kim SH. Sphingosine-1-Phosphate: Biomarker, Contributor, or Target for Asthma? Allergy Asthma Immunol Res . 2019;11:299-301.

28. Ban GY, Youn DY, Ye YM, Park HS. Increased expression of serine palmitoyl transferase and ORMDL3 polymorphism are associated with eosinophilic inflammation and airflow limitation in aspirin-exacerbated respiratory disease. PLoS One . 2020;15:e240334.

29. Oyeniran C, Sturgill JL, Hait NC, et al. Aberrant ORM (yeast)-like protein isoform 3 (ORMDL3) expression dysregulates ceramide homeostasis in cells and ceramide exacerbates allergic asthma in mice. $J$ Allergy Clin Immunol . 2015;136:1035-1046.

30. Petrache I, Kamocki K, Poirier C, et al. Ceramide synthases expression and role of ceramide synthase-2 in the lung: insight from human lung cells and mouse models. PLoS One . 2013;8:e62968. 
31. Kim SH, Jung HW, Kim M, et al. Ceramide/sphingosine-1-phosphate imbalance is associated with distinct inflammatory phenotypes of uncontrolled asthma. Allergy . 2020.

32. Wang M, Zhang N, Zheng M, et al. Cross-talk between TH2 and TH17 pathways in patients with chronic rhinosinusitis with nasal polyps.J Allergy Clin Immunol . 2019;144:1254-1264.

33. Na H, Lim H, Choi G, et al. Concomitant suppression of TH2 and TH17 cell responses in allergic asthma by targeting retinoic acid receptor-related orphan receptor gammat. J Allergy Clin Immunol . 2018;141:2061-2073 e2065.

34. Mastalerz L, Tyrak KE. Biomarkers for predicting response to long-term high dose aspirin therapy in aspirin-exacerbated respiratory disease. Clinical and translational allergy . 2021;11:e12048.

35. Larche M. Regulatory T cells in allergy and asthma. Chest . 2007;132:1007-1014.

36. Ben Mkaddem S, Benhamou M, Monteiro RC. Understanding Fc Receptor Involvement in Inflammatory Diseases: From Mechanisms to New Therapeutic Tools. Front Immunol . 2019;10:811.

37. Gomez G. Current Strategies to Inhibit High Affinity FcepsilonRI-Mediated Signaling for the Treatment of Allergic Disease.Front Immunol . 2019;10:175.

38. Wesolowska-Andersen A, Seibold MA. Airway molecular endotypes of asthma: dissecting the heterogeneity. Curr Opin Allergy Clin Immunol . 2015;15:163-168.

39. Gelardi M, Iannuzzi L, Quaranta N, et al. NASAL cytology: practical aspects and clinical relevance. Clin Exp Allergy . 2016;46:785-792.

40. Heffler E, Landi M, Caruso C, et al. Nasal cytology: Methodology with application to clinical practice and research. Clin Exp Allergy . 2018;48:1092-1106.

41. Poole A, Urbanek C, Eng C, et al. Dissecting childhood asthma with nasal transcriptomics distinguishes subphenotypes of disease. J Allergy Clin Immunol . 2014;133:670-678 e612.

Table 1. Demographic data of the study subjects

\begin{tabular}{llll}
\hline & AERD $(\mathrm{n}=5)$ & ATA $(\mathrm{n}=5)$ & $P$ value \\
\hline Age (yr) & $55.2 \pm 9.8$ & $47.0 \pm 7.3$ & 0.310 \\
Sex (Female) & $4(80 \%)$ & $5(100 \%)$ & 0.292 \\
Atopy & $2(40 \%)$ & $2(40 \%)$ & 1.000 \\
Chronic rhinosinusitis & $3(60 \%)$ & $4(80 \%)$ & 1.000 \\
Total IgE (KU/L) & $407.8 \pm 484.7$ & $250.2 \pm 352.0$ & 0.222 \\
Sputum eosinophil (\%) & $74.3 \pm 30.0$ & $14.8 \pm 18.8$ & 0.032 \\
Peripheral eosinophil count $(/ \mu \mathrm{L})$ & $511.11 \pm 317.98$ & $800.00 \pm 492.16$ & 0.151 \\
FeNO (ppb) & $72.6 \pm 52.4$ & $46.6 \pm 18.1$ & 0.841 \\
Baseline FEV $1 \%$ Pred) & $70.3 \pm 14.3$ & $77.4 \pm 18.4$ & 0.548 \\
Baseline FEV $/$ FVC & $70.4 \pm 9.7$ & $77.0 \pm 11.1$ & 0.421 \\
Baseline MMEF (\% Pred) & $34.4 \pm 9.8$ & $43.6 \pm 16.9$ & 0.548 \\
Severe asthma & $4(80 \%)$ & $5(100 \%)$ & 0.292 \\
\hline
\end{tabular}

Values are given as n (\%) for categorical variables and as mean \pm SD for continuous variables. $P$ values were analyzed by the Mann Whitney test and Fisher's exact test. $A E R D$, aspirin-exacerbated respiratory disease; $A T A$, aspirin tolerant asthma; $F e N O$, fractional exhaled nitric oxide; $F E V 1$, forced expiratory volume in 1 second; $F V C$, forced Vital Capacity; $M M E F$, maximum midexpiratory flow.

Table 2. Top 19 candidate differentially correlated genes significantly different between AERD and ATA 


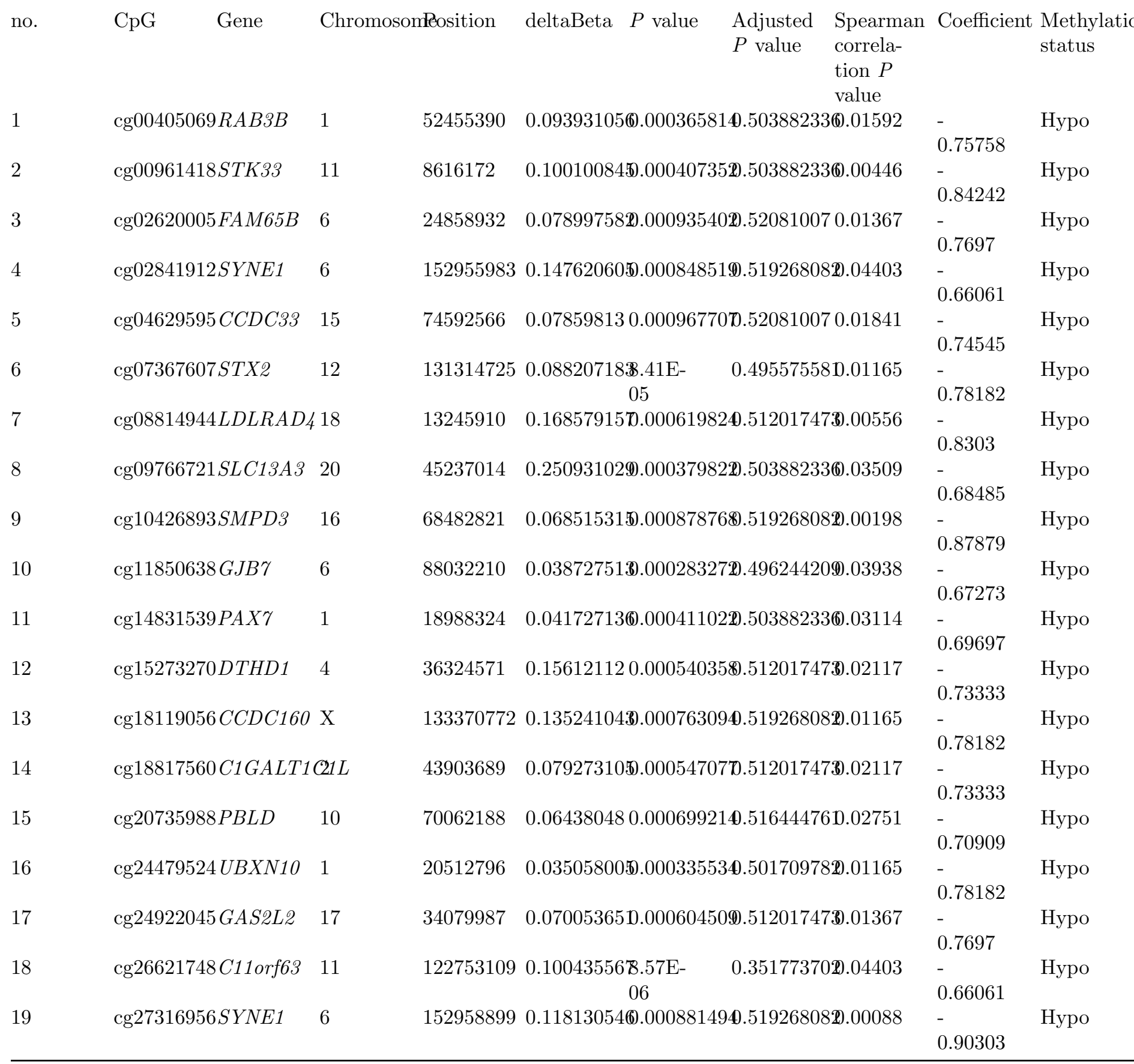

\section{Figure legends}

Figure 1. Gene expression signatures between patients with AERD and those with ATA. (A) Heatmap, (B) Scatter plot (C) Volcano plot for DEGs.

AERD, aspirin-exacerbated respiratory disease; ATA, aspirin tolerant asthma; DEG, differentially expressed gene

Figure 2. Genome-wide methylation profiling of DMR. (A) Pheatmap of EPIC (top 1,000 variable CpGs) 
according to aspirin intolerance. (B) Genomic distribution of DNA methylation changes in AERD compared to ATA by genomic features.

$1^{\text {st }}$ Exon: first exon region, 3'UTR: 3-prime untranslated region, 5'UTR: 5-prime untranslated region, Body: gene body, ExonBnd: exon boundaries, IGR: intergenic region, TSS200: region from transcription start site (TSS) to $-200 \mathrm{nt}$ upstream of TSS, TSS1500: -200 to -1500 nt upstream of TSS.

Figure 3. Heatmap of DCG pairs having an inverse correlation between expression and methylation.

DCG, differentially correlated gene.

Figure 4. Gene connection (A) and signaling pathways with $\mathrm{T}$ helper cell differentiation/activation (B) and Fc Epsilon RI signaling (C). The bottom panel provides a legend of the IPA network.

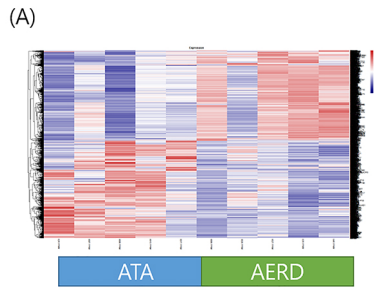

(B)

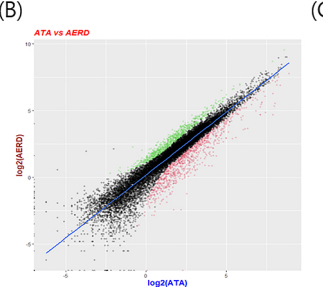

(C)

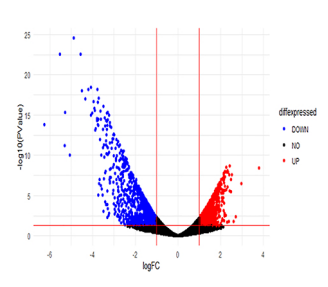

(A)

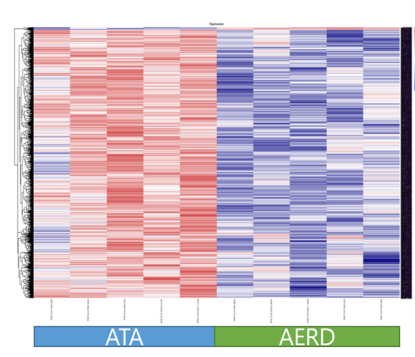

(B)
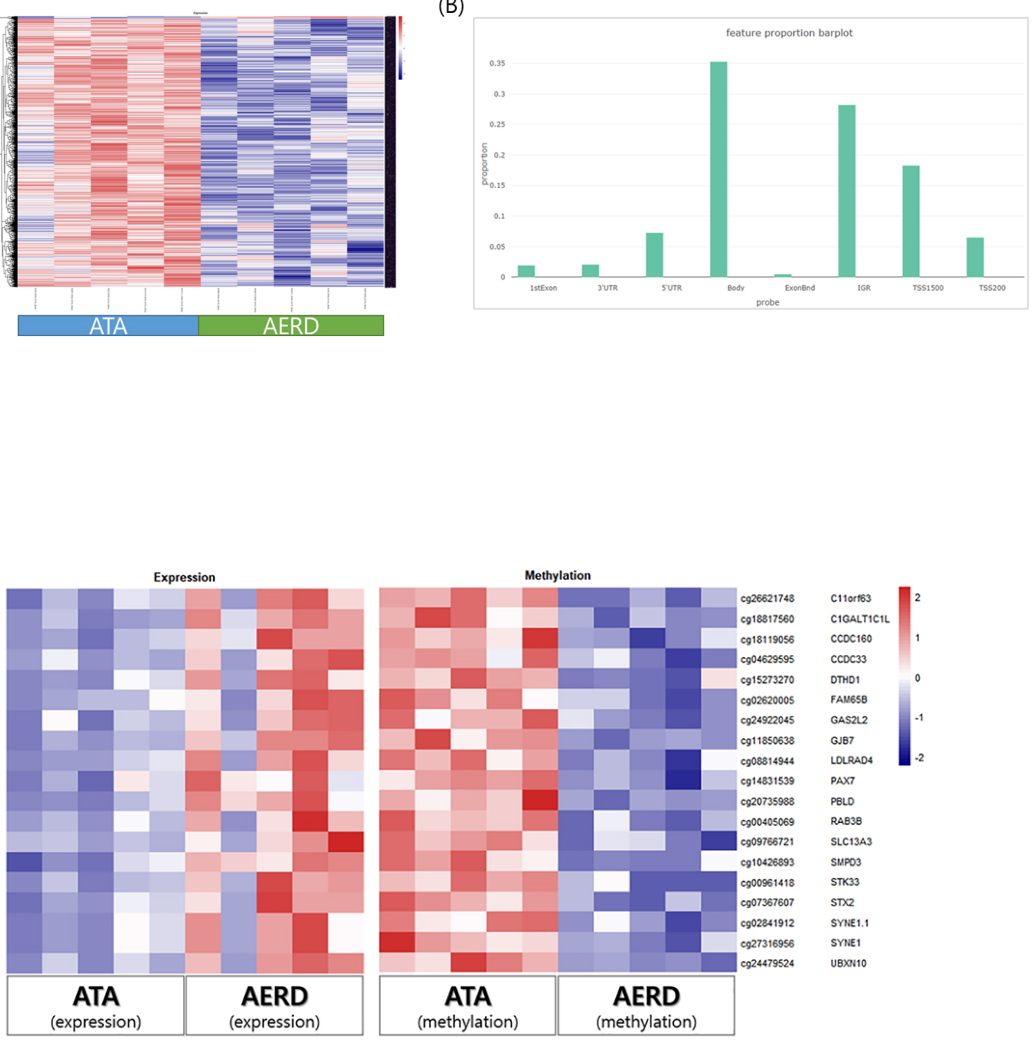


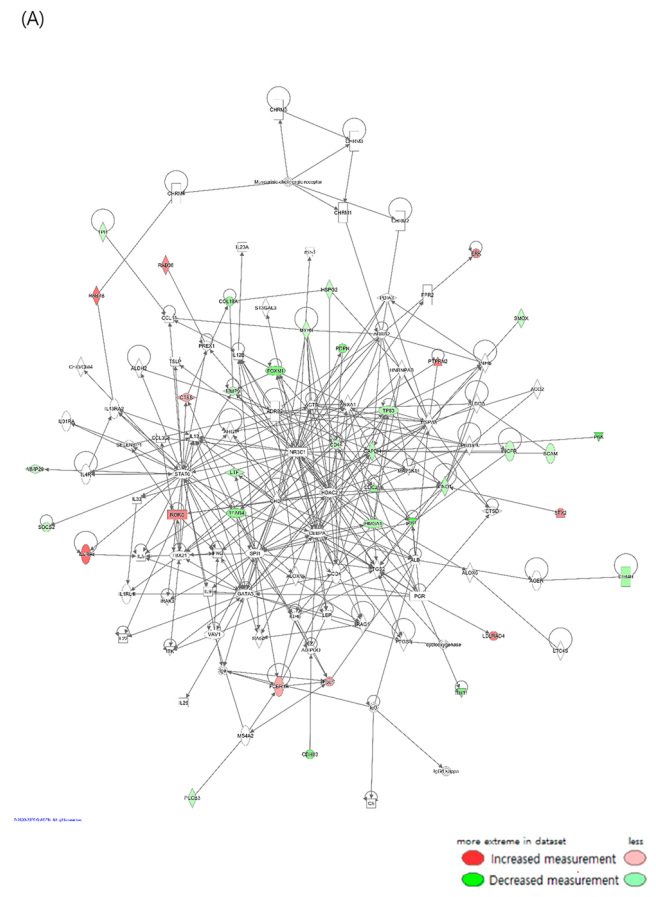

(B)
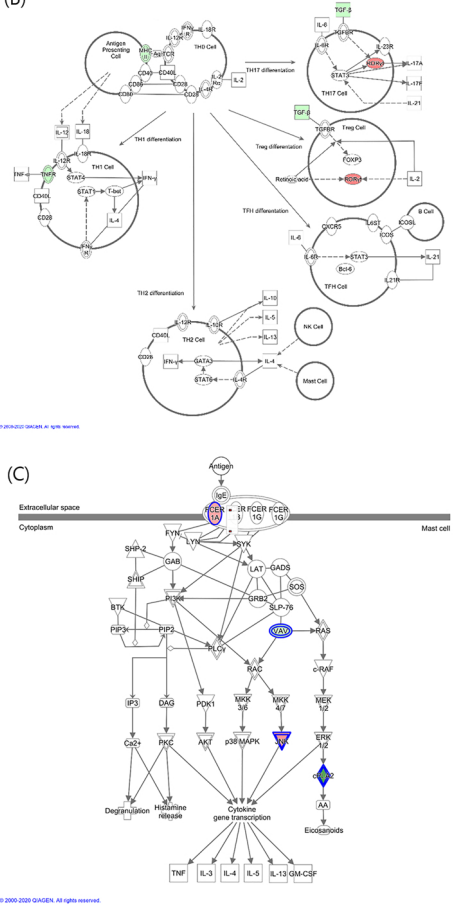